\title{
Calibrating and preserving the energy scale of the Tile Calorimeter cells during four years of LHC data-taking
}

\author{
Emmanuelle Dubreuil on behalf of the ATLAS Collaboration, Laboratoire de Physique Corpusculaire de \\ Clermont Ferrand, CNRS/IN2P3, France
}

\begin{abstract}
TileCal is the hadronic calorimeter covering the most central region of ATLAS experiment at the LHC. This sampling calorimeter uses iron plates as absorber and plastic scintillating tiles as the active material. Scintillation light produced in the tiles is transmitted by wavelength shifting fibers to photomultipliers tubes (PMTs). The resulting electronic signals from the approximatively 10000 PMTs are measured and digitized every $25 \mathrm{~ns}$ before being transferred to off-detector data-acquisition systems. A set of calibration systems allow to monitor and equalize the calorimeter at each stage of the signal production, from scintillation light to digitization. This calibration suite is based on signal generation from different sources: A Cs radioactive source, laser light, charge injection and charge integration over thousands of bunch crossings of minimum bias events produced in proton-proton collisions. This contribution presents a brief description of the different TileCal calibration systems and their performances. It elaborates on the methodology of using and combining the individual systems measurements to preserve the calorimeter cell energy scale stable at the \% level. The contribution to the LHC luminosity monitoring and measurement is also discussed.
\end{abstract}

Index Terms-ATLAS, Calibration, LHC, Tile Calorimeter

\section{INTRODUCTION}

$\mathbf{T}$ he Tile Calorimeter (TileCal) is the hadronic calorimeter of the ATLAS experiment [1] installed at the Large Hadron Collider (LHC) at CERN (Figure 1). It contributes to the measurement of the missing transverse energy, hadrons, taus and jets. It provides also input signal to the Level 1 Trigger. TileCal is a sampling device made of layers of absorber material, iron, and of layers of active material, scintillating plastic tiles. Hadronic showers developed in the TileCal create light in the plastic tiles. This light is collected by wavelength shifting fibers and sent to photomultipliers (PMTs). The charge collected by each PMT is then digitized and passed to the ATLAS read-out system. Cells are defined by grouping these fibers in bundles and coupling them to a same PMT. The signals from the PMTs are shaped then amplified. Two gains are used (so-called low and high gains), the gain ratio is 1:64. Each PMT has a seperate integrator system which integrates the PMT current over time. The calibration systems provide a complete diagnosis of the hardware chain, from the active modules to the read-out electronics.

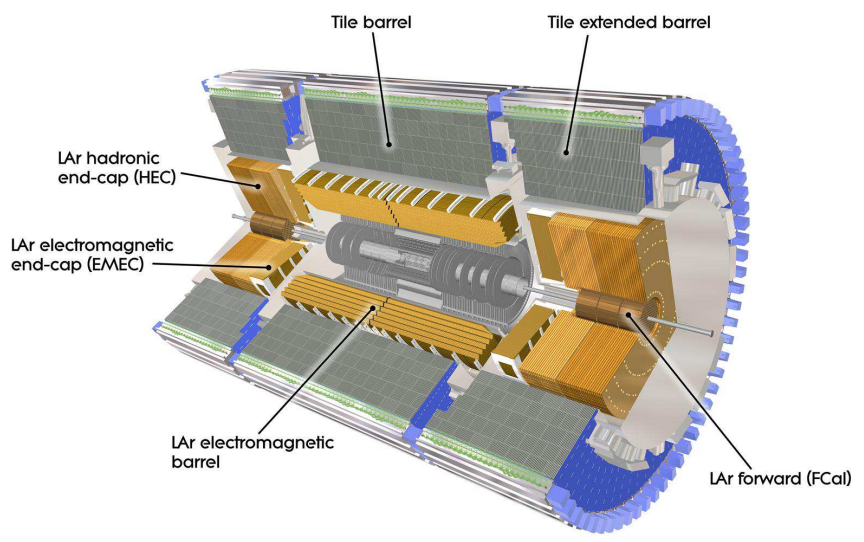

Fig. 1. Calorimetric system of ATLAS detector

\section{The TILE CALORIMETER}

The Tile Calorimeter [2] uses scintillating plastic tiles as its active material and iron as absorber. It consists of one long barrel (covering $0<|\eta|<0.8$ ) and two extended barrels $(0.8$ $<|\eta|<1.7)$, as shown on Figure 1. The inner and outer radii are 2.23 and $4.23 \mathrm{~m}$ respectively. The gap between long and extended barrels is filled with cables, services and power supplies for the other sub-detectors of ATLAS. For this reason, scintillating tiles have been placed here to act as the active material for the non-instrumented regions (so-called gap/crack scintillators). The long and extended barrels are divided azimuthally into 64 modules. The modules are installed radially around the beam axis to get almost full coverage in $\phi$. The $3 \mathrm{~mm}$ thick tiles are placed perpendicularly to the beam pipe. The read-out is also segmented longitudinally in three layers in both long and extended barrels (called A, B and D), as shown on Figure 2. The cell granularity is $\Delta \eta \times \Delta \phi=0.1 \times 0.1$ for the first two layers and $\Delta \eta \times \Delta \phi=0.1 \times 0.2$ for the third one.

\section{Monitoring And CAlibrations of the Tile CALORIMETER}

The calibration systems are important to adjust the energy channel by channel. Three systems exist to calibrate the TileCal (Figure 3) :

\footnotetext{
e-mail: emmanuelle.dubreuil@cern.ch.
} 


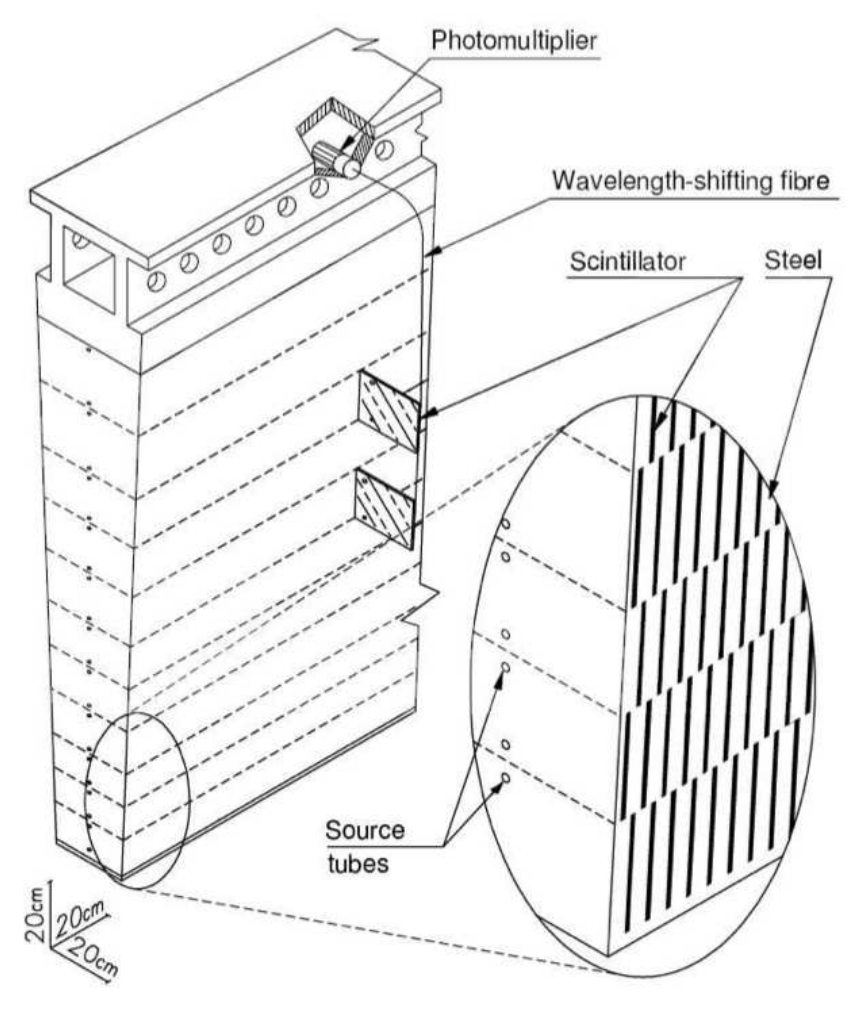

Fig. 2. Structure of a module of the Tile Calorimeter

- The Cesium system : for the calibration of the tile optic components and photomultipliers

- The Laser system : for the calibration of the photomultiplier gains and linearities

- The Charge Injection system : for the calibration of the front-end electronics

These three systems together allow to calibrate all the parts of the TileCal and determine the origin of any issue.

The final cell energy is expressed as follows :

$\mathrm{E}[\mathrm{GeV}]=\mathrm{A}[\mathrm{ADC}] \cdot C_{A D C \rightarrow p C} \cdot C_{\text {laser }} \cdot C_{C s} \cdot C_{p C \rightarrow G e V}$

where $\mathrm{A}[\mathrm{ADC}]$ is the pulse amplitude in $\mathrm{ADC}$ counts. The last factor $C_{p C \rightarrow G e V}$ is used to convert charge to electromagnetic scale energy. It was determined from the test beam measurements in 2001-2003 using electron and muon beams. The other constants are determined with each calibration system, which monitor the read-out electronics, the PMTs and the optics (scintillators plus wavelength shifting fibers). These systems are described in the following sections.

\section{A. The Cesium system}

The cesium calibration is done by using a movable radioactive source of ${ }^{137} \mathrm{Cs}$ which induces light which passes through scintillator tiles by the same mechanisms as particles from LHC interactions. This calibration is taken about once a month, because a cesium scan lasts for 6 hours. They are typically taken during technical stops or machine developments of the LHC. This source has an activity of around $330 \mathrm{MBq}$, and emits $662 \mathrm{keV} \gamma$-rays. They are hydraulically

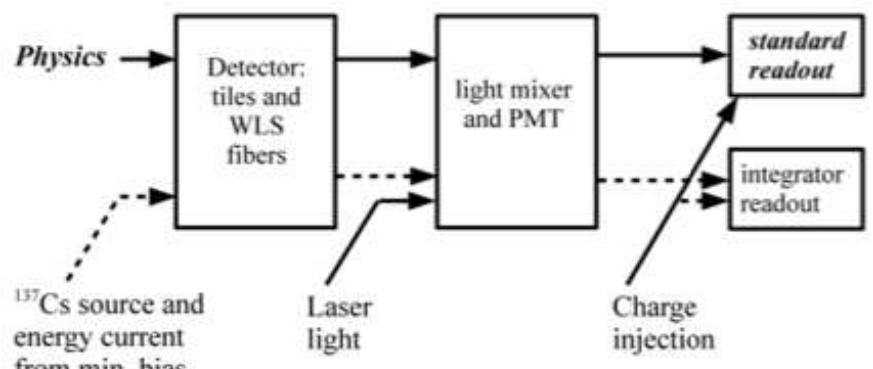

from min. bias

Fig. 3. Tile Calorimeter hardware calibration system

Drift in March-November 2012

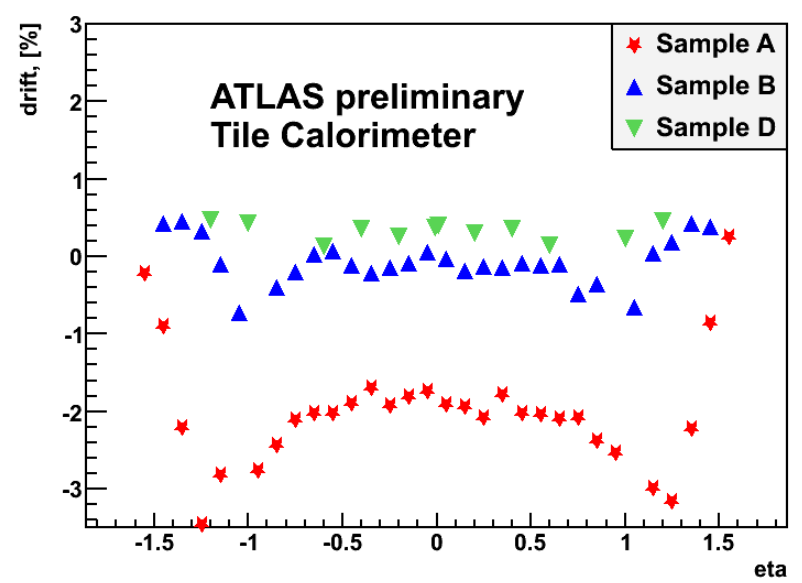

Fig. 4. Drift of the signal, measured by the Cesium system, between March and November 2012 for the three layers of the Tile Calorimeter

driven through a system of steel tubes passing through each of the cells. The integrator circuit measures the charge from the PMT as the source traverses the connected cell. The total integrated current is normalized to the cell size. The cesium system calibrates the entire hardware chain and provides an absolute scale determination. Using a moveable gamma source allows to check the quality of the optical response and its uniformity, to equalize the response of all read-out cells by adjusting the HV of the corresponding PMT to obtain the same average current from each cell and to monitor in time the average current of each cell by running the source again. The Cesium calibration allows to test the optical chain with precision better than $0.3 \%$. The pseudo-rapidity dependance of the detector response drift, determined by the Cesium system, over the 2012 run is shown in Figure 4.

\section{B. The Laser system}

The Laser calibration system is dedicated to the monitoring and the calibration of the gain and the linearity of each TileCal PMT between two Cesium scans. It is also used for timing studies. The laser emits a beam of $532 \mathrm{~nm}$ light. The wavelength of the laser light is close to the typical wavelenght of the light produced by the physical signals. Using a light distribution system, the initial beam is split into optical fibers 


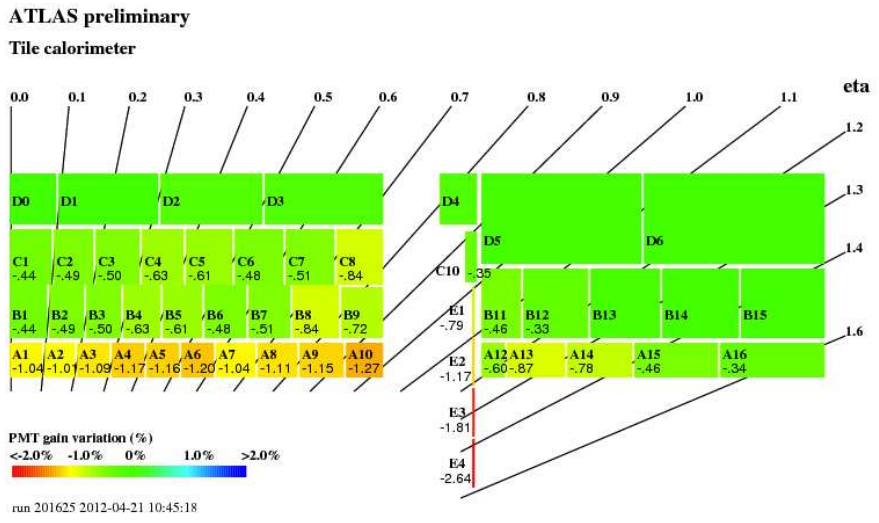

Fig. 5. Mean gain variation (in \%) in the PMTs of the Tile Calorimeter, between the $19^{t h}$ March 2012 and the $21^{\text {st }}$ April 2012 (between 2 consecutive Cesium scans)

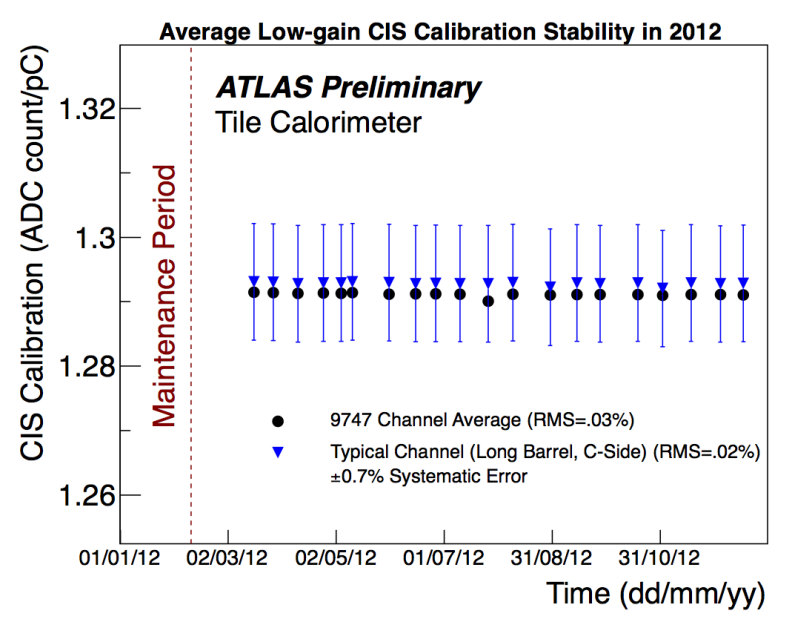

Fig. 6. Time stability of the average low-gain read-out calibration constants from February 2012 to December 2012

and the light is sent to all the TileCal PMTs. The amplitude of light pulses is monitored using a set of photodiodes monitored by a moveable $\alpha$-source. The Laser calibration is taken twice a week. It aims at equalizing the response of the TileCal channels in time, taking as reference the latest Cesium scan. Laser pulses are also emitted during empty bunch crossings, during the physics runs, in order to monitor the timing of the channels responses. For a period of one month, the typical precision of the laser system is better than $0.5 \%$ on the gain variation (Figure 5).

\section{The Charge Injection system}

The Charge Injection System (CIS) is a feature of the TileCal front-end electronics that simulates physics signals in the channels by injecting a known charge and measuring the electronic response. It is used jointly with the laser and cesium calibrations. There are two precision capacitors for each channel, discharging a well defined charge in the high or low gain $\mathrm{ADC}$ of the channel, respectively. This amounts to 19704 ADCs to calibrate. It is used to measure the pC/ADC

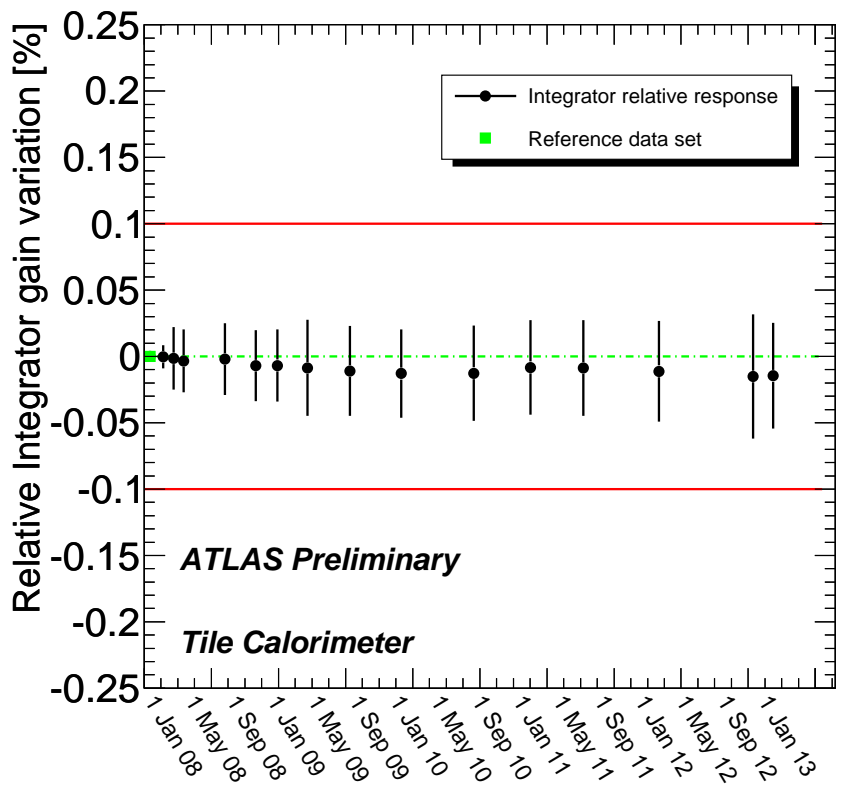

Fig. 7. Relative variation of the integrator gain used by the Cesium calibration system obtained by comparing the gains of all the channels of the Tile Calorimeter

conversion factor for the digital read-out of physics and laser calibration data. CIS calibration factors are calculated from dedicated charge injection runs and are used for monitoring purposes and for correcting energy reconstruction. The precision of the CIS calibrations is within $0.7 \%$ (Figure 6).

\section{The Integrator system}

In the high energy proton-proton collisions at the LHC, the dominating interactions are soft parton interaction, also called Minimum Bias (MB) events, which are uniformly distributed around the interaction point. During Cesium scans, the integrator systems measure the response to the passing source, while during physics runs with collisions, it measures the detector response to the MB events. The electronic board plugged just after every PMT has a signal integrator with an integration time ranging between $10-20 \mathrm{~ms}$, depending on its configuration. This signal is continuous over time which enables the integrator to monitor the response of all the TileCal cells during data-taking. This system provides a $0.5 \%$ precise monitoring for the response of one single cell of the TileCal (Figure 7). Moreover, dedicated studies show a linear dependance between MB signal and the instantaneous luminosity delivered by the LHC. This device and other ATLAS luminosity monitors demonstrates consistency of the luminosity measurement in ATLAS experiment.

\section{Performances}

\section{A. Combined calibrations}

Without collisions, an updrift of the TileCal channels gains is observed. This effect disappeared in 2011 when the protonproton collisions started at higher luminosity than previously 


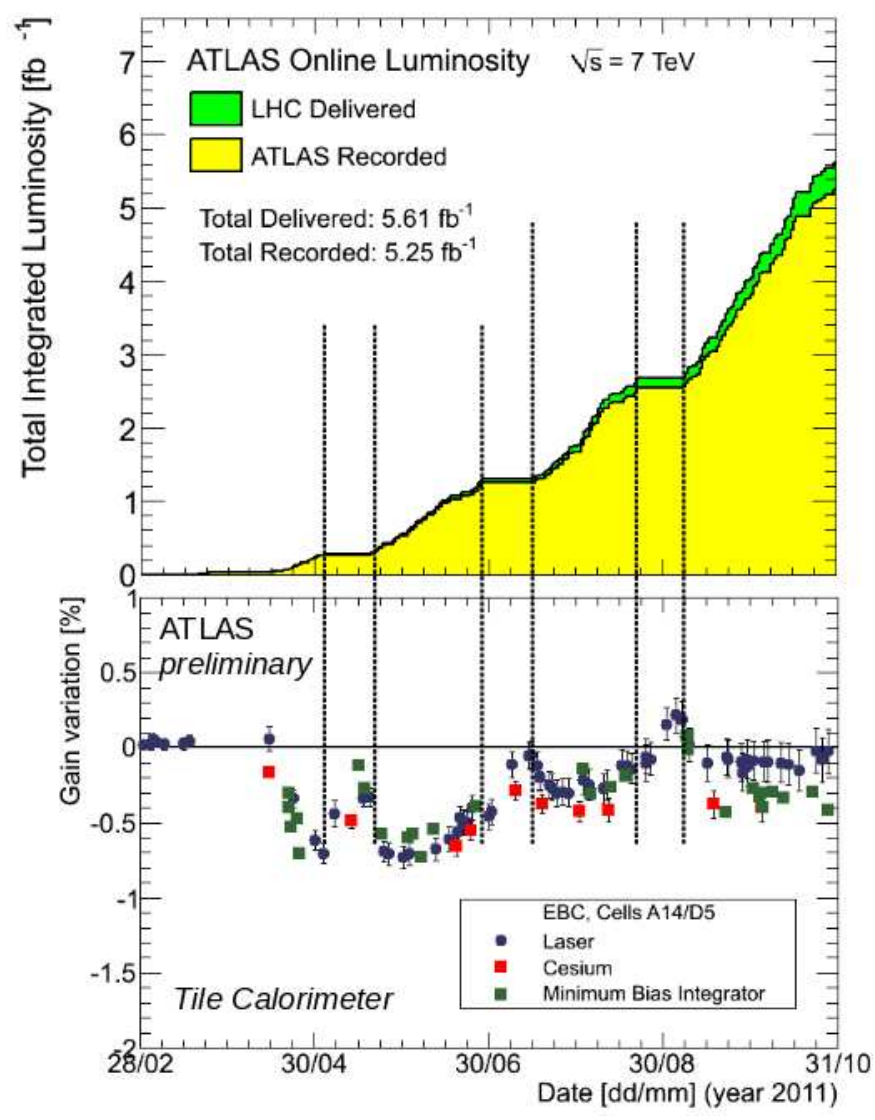

Fig. 8. The evolution of ATLAS total integrated luminosity (top) and the evolution of the response of a cell at low radius. The response is measured separately by the Cesium, Laser and Integrator calibration systems

as shown on Figure 8. Then a downdrift is observed during the collision periods, and a slow recovery during the maintenance periods. This effect is seen simultaneously by the Cesium, the Laser and the integrator systems. The fact that all the three systems show the same behavior indicates that it is caused by a drift of the PMT gains. It is mostly affecting PMTs at lower radius which are the channels connected to the cells receiving the largest number of particles.

\section{B. Monitoring of the High Voltages (HV) and temperature}

The gain of the PMTs is fixed to a precise value, by applying High Voltage (HV). The HV have to be kept stable (within $0.5 \mathrm{~V}$ ) to maintain the energy measurement as stable as possible.

During the whole 2012-2013 run, the gain computed from the $\mathrm{HV}$ is stable within $0.05 \%$, as it shows on the Figure 9. Moreover, some comparisons of the gain computed by the Laser system, the Cesium system and the HV have been done to diagnose some pathological channels.

The PMT gain is also a function of temperature, therefore it needs to be monitored (see Figure 10). Technically, the temperature is measured in 7 different points in a module (in different eletronic cards, on one PMT). The results showed that a variation of $1^{\circ}$ Celsius induces a gain variation of 0.2 $\%$.

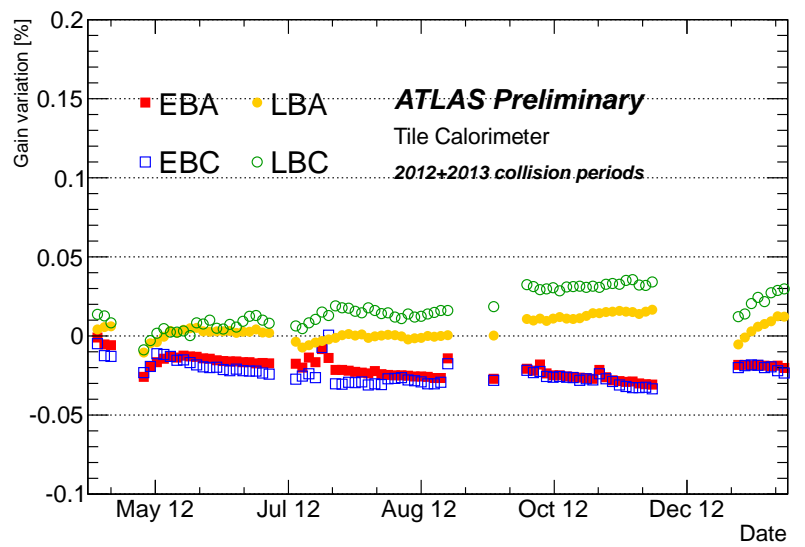

Fig. 9. Evolution of the PMT gain computed from the HV for the four partitions of the Tile Calorimeter

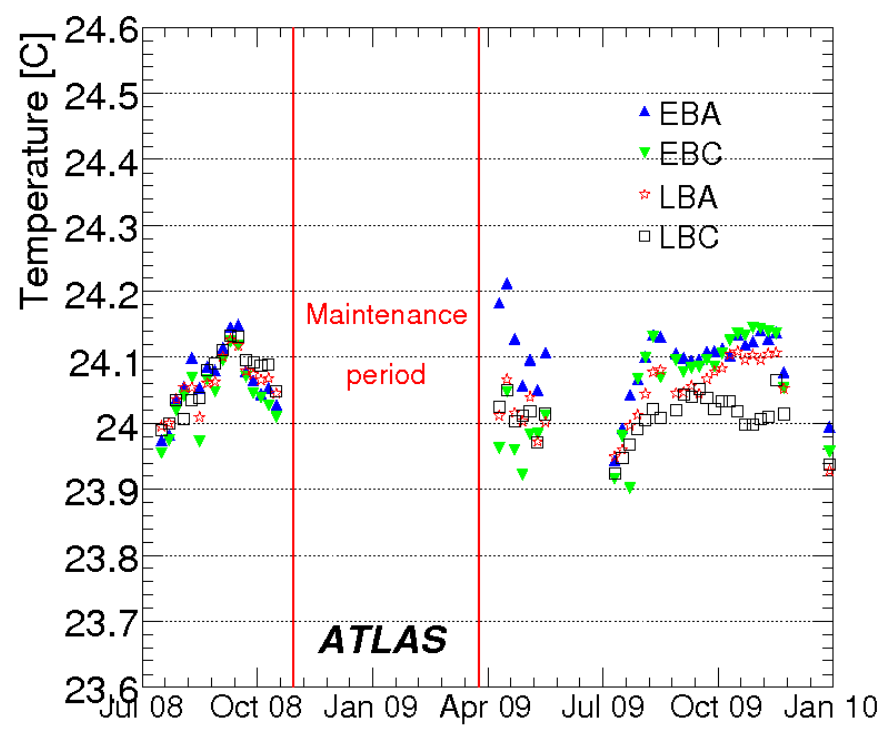

Fig. 10. Stability of the temperature, averaging over all drawers between July 2008 and January 2010

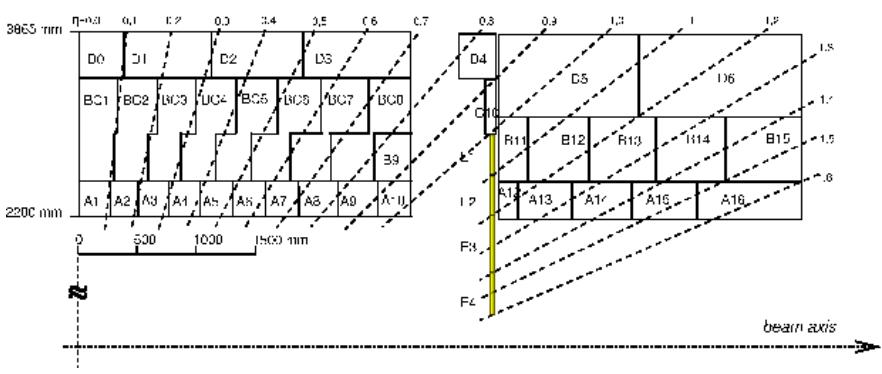

Fig. 11. Schema of the gap and crack scintaillators in the Tile Calorimeter

\section{Calibration of Gap-Crack cells}

The gap scintillators (Figure 11), E1 and E2 cells, are monitored by the Laser and Cesium system but are calibrated 


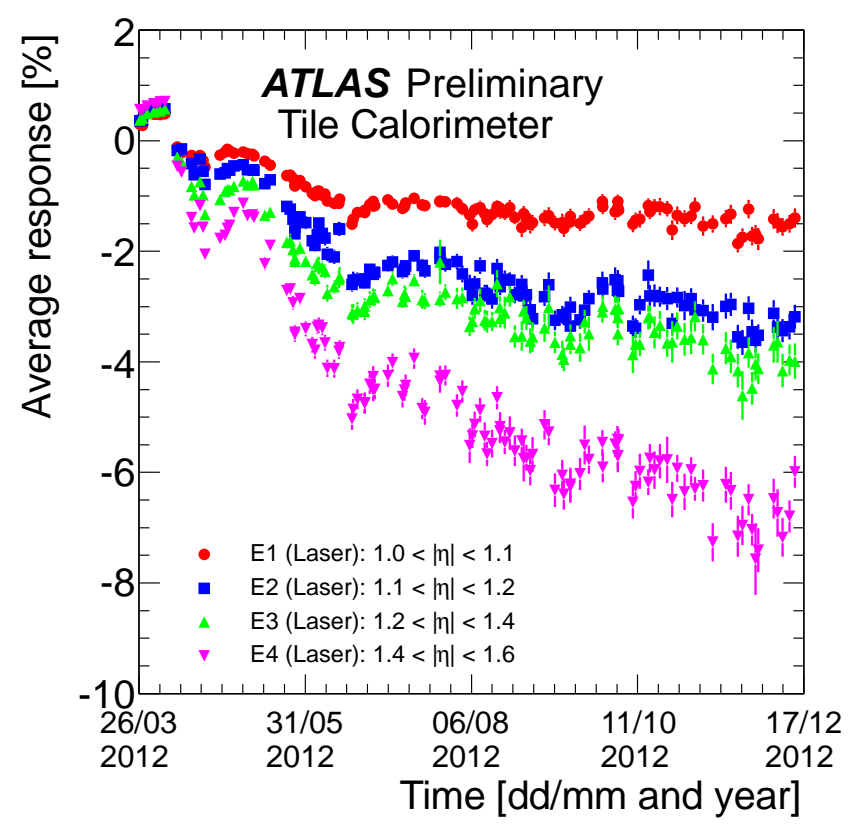

Fig. 12. Evolution of the mean response of the E1, E2, E3 and E4 cells, measured with the Laser calibration system

only with the Cesium system. In 2012, a maximal downdrift of $-3 \%$ is observed for only the PMTs, measured by the Laser, as shown on the Figure 12. The crack scintillators (Figure 11), E3 and E4 cells, are monitored and calibrated by the Laser system and studies using muons from collisions (there is no Cesium calibration in these cells). With the Laser calibration, a maximal downdrift of $-8 \%$ is observed for all the year 2012 (Figure 12).

\section{CONCLUSION}

The calibration systems of the ATLAS Tile Calorimeter have been presented. The Cesium, Laser and Charge Injection systems calibrate and monitor the calorimeter response with a $0.3-1.0 \%$ precision. Moreover, the integrator system provides a $0.5 \%$ precise monitoring for the response of one cell. When combining results from all calibration systems one can determine what causes changes in the overall detector response.

\section{ACKNOWLEDGMENT}

I would like to thank the ATLAS Tile Community for all their support and expertise. Moreover I would like to thank the LPC and the CNRS/IN2P3 for financing my attendance to the conference and the Region Auvergne for financing my thesis.

\section{REFERENCES}

[1] ATLAS Collaboration, The ATLAS Experiment at the CERN Large Hadron Collider, JINSTS08003(3)2008.

[2] ATLAS Collaboration, ATLAS Tile Calorimeter Technical Design Report, CERN-LHCC-96-421996. 